Ann. Génét. Sél. anim., I969, 1 (4), 341-348.

\title{
EXAMENS MORPHOLOGIQUES, CARYOLOGIQUES, PHYSIOLOGIQUES ET PATHOLOGIQUES DE BOUCS STÉRILES SANS CORNES
}

\author{
J.-M. CORTEEL, Françoise HULOT*, M. COUROT, J. ATTAL \\ et A. PHILIPPON** \\ Laboratoire de Physiologie de la Reproduction, \\ Centre de Recherches de Tours, 37-Nouzilly \\ $\left({ }^{*}\right)$ Station centrale de Génétique animale, \\ Centre national de Recherches zootechniques, 78-Jouy-en-Josas \\ (**) Laboratoire de Pathologie de la Reproduction, \\ Centre de Recherches de Tours, 37-Nouzilly \\ Institut national de la Recherche agronomique
}

\section{RÉSUMÉ}

Huit boucs sans cornes réputés stériles ont été examinés. Un animal atteint d'hypoplasie visible des organes génitaux externes était de sexe génétique femelle XX. Les 7 autres boucs étaient de sexe génétique mâle XY. Cinq d'entre eux présentaient des atteintes caractérisées de l'épididyme. Trois de ces cinq boucs présentaient également des atteintes caractérisées du testicule. Chez les deux derniers animaux de sexe chromosomique XY, la stérilité n'était pas expliquable à l'examen du tractus génital.

\section{INTRODUCTION}

En I968 et en I969, 8 boucs sans corne réputés stériles ont été l'objet d'examens morphologiques et caryologiques. Cinq d'entre eux ont été soumis à des examens anatomiques, physiologiques et pathologiques plus étendus.

\section{I. - ASPECT EXTÉRIEUR DES ANIMAUX}

Un bouc $(A)$ présentait des caractères sexuels secondaires mâles très peu développés (barbiche, encolure, fig. I, A) et un appareil génital externe de dimensions anormalement réduites (scrotum en particulier, fig. I, B); tous les autres animaux ( $B$ à $H$ ) avaient 1 'aspect extérieur de boucs apparemment normaux (tabl. I). 
TABLEAU I

Caryotype, Morphologie et Anatomie génitale de 8 boucs stériles sans cornes

\begin{tabular}{|c|c|c|c|c|c|c|}
\hline Boucs & $\begin{array}{c}\text { No } \\
\text { Oreille }\end{array}$ & $\begin{array}{l}\text { Date } \\
\text { naissance } \\
\text { et âge } \\
\text { abattage }\end{array}$ & Race & $\begin{array}{l}\text { Sexe } \\
\text { chromo- } \\
\text { somique }\end{array}$ & $\begin{array}{l}\text { Aspect extérieur } \\
\text { des animaux }\end{array}$ & Organes génitaux \\
\hline$A$ & 7979 & $\begin{array}{c}6-5-67 \\
22 \text { mois }\end{array}$ & $\begin{array}{c}\text { alpine } \\
\text { chamoisée }\end{array}$ & $\mathrm{xx}$ & $\begin{array}{l}\text { Caractères sexuels } \\
\text { secondaires peu } \\
\text { marqués, orga- } \\
\text { nes génitaux peu } \\
\text { développés }\end{array}$ & $\begin{array}{l}\text { Testicules et épididymes } \\
\text { infantiles }\end{array}$ \\
\hline$B$ & 0527 & $\begin{array}{l}23-2-68 \\
\text { I3 mois }\end{array}$ & $\begin{array}{l}\text { alpine } \\
\text { chamoisée }\end{array}$ & $\mathrm{XY}$ & Mâle normal & $\begin{array}{l}\text { Testicules : granules de } \\
\text { calcification } \\
\text { Epididymes : induration } \\
\text { et obstruction bila- } \\
\text { térale }\end{array}$ \\
\hline C & 7975 & $\begin{array}{l}5-2-67 \\
25 \text { mois }\end{array}$ & poitevine & $\mathrm{XY}$ & Mâle normal & Idem \\
\hline$D$ & 7976 & Inconnue & Sâanen & $X Y$ & Mâle normal & Idem \\
\hline$E$ & 7980 & $\begin{array}{l}\text { I } 3-1-67 \\
\text { I mois }\end{array}$ & $\begin{array}{c}\text { alpine } \\
\text { chamoisée }\end{array}$ & $\mathrm{XY}$ & Mâle normal & R.A.S. \\
\hline$F$ & 7978 & $\begin{array}{l}28-1-68 \\
\text { I } 4 \text { mois }\end{array}$ & $\begin{array}{c}\text { alpine } \\
\text { chamoisée }\end{array}$ & $\mathrm{XY}$ & Mâle normal & $\begin{array}{l}\text { Induration et obstruc- } \\
\text { tion bilatérale de } \\
\text { l'épididyme }\end{array}$ \\
\hline G & 8238 & Inconnue & Sâanen & $\mathrm{XY}$ & Mâle normal & Idem \\
\hline$H$ & 8237 & Inconnue & Sâanen & $X Y$ & Mâle normal & R.A.S. \\
\hline
\end{tabular}

\section{II. - SEXE CHROMOSOMIQUE DES ANIMAUX}

L'examen des chromosomes, réalisé à partir de cultures de sang total (microtechnique de DE GRouchy et al., I964) a révélé que 1'individu A dont les organes génitaux étaient peu développés était de génotype femelle (caryotype $\mathrm{XX}$ ) et que

Planche I. - Animal $A$, âgé de 22 mois, de caryotype $X X$

A : Tête et encolure : à noter la finesse de l'encolure et de la barbiche.

B : Région anogénitale : on remarque l'ébauche d'un sillon vulvaire et le faible développement du scrotum.

C : Testicule et épididyme : leur très petite taille est voisine de celle des organes d'un chevreau peu après la naissance.

D : Carrefour urogénital après ablation des structures sexuelles mâles normales (canaux déférents, ampoules déférentes, vésicules séminales et naissance du pénis) : on remarque les uretères et plusieurs structures supplémentaires non identifiées qui pourraient être d'origine femelle. 
HUIT BOUCS STÉRILES SANS CORNES
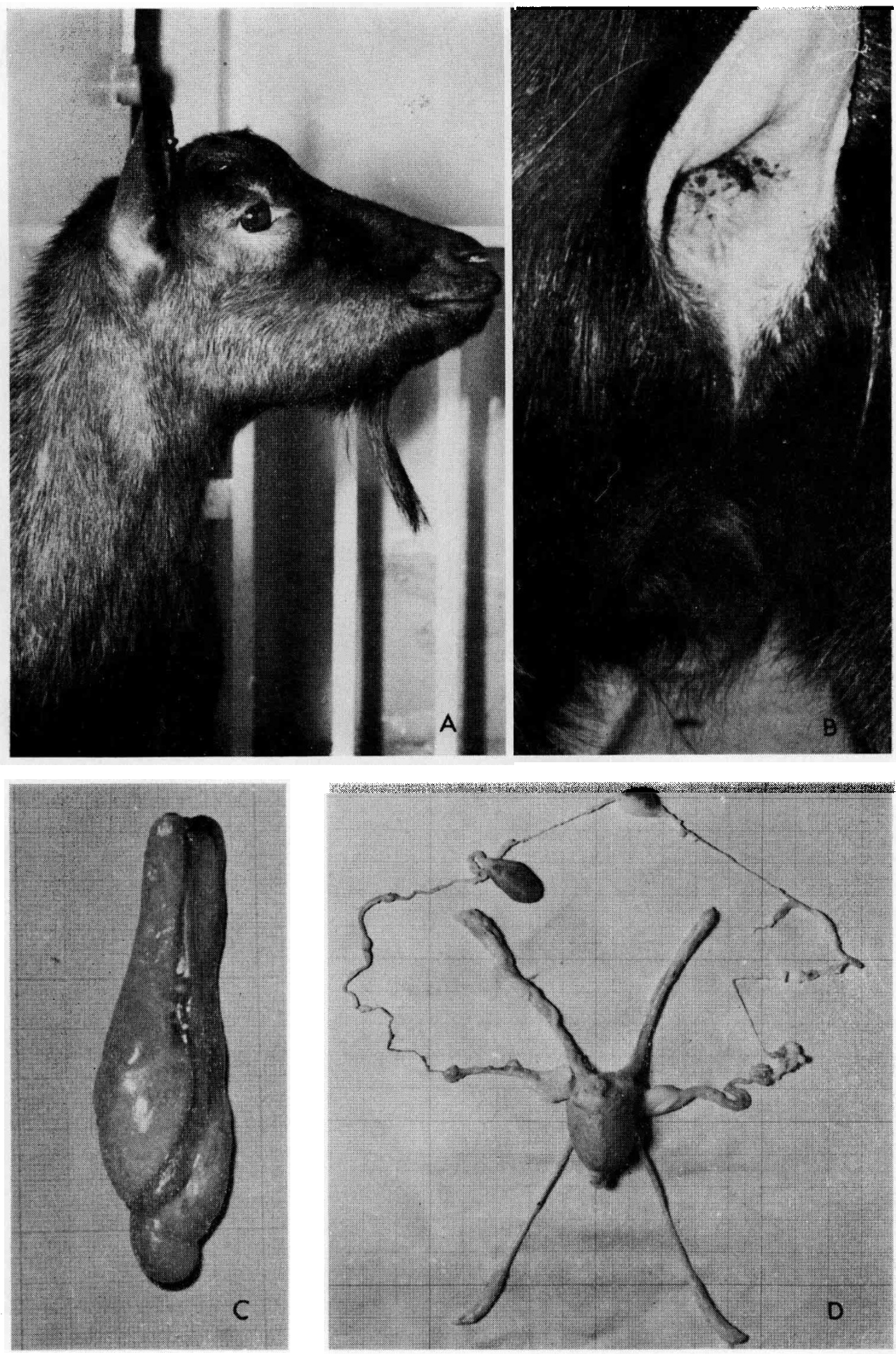

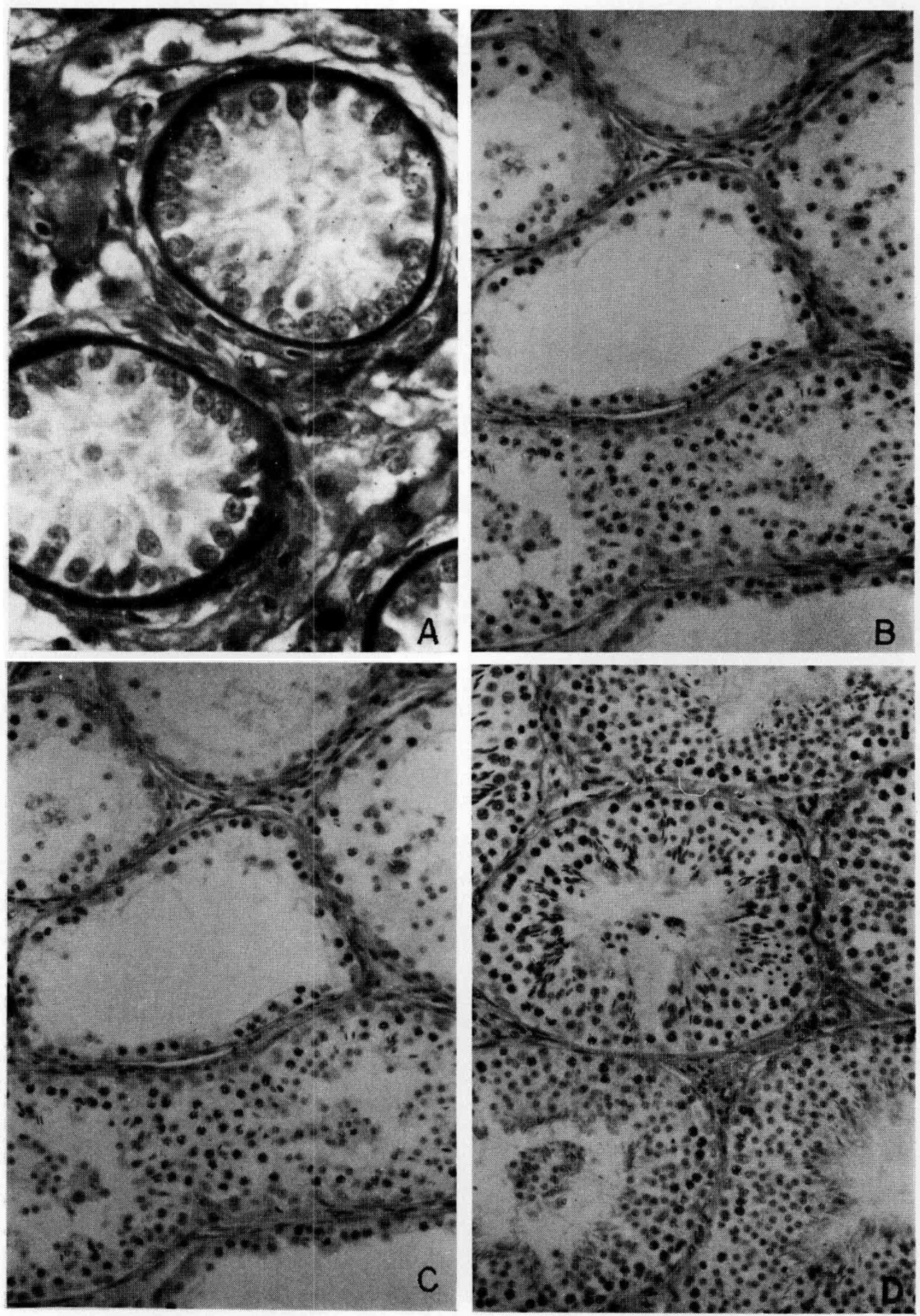
les animaux de phénotype normal $(B$ à $H$ ) étaient génétiquement mâles (caryotype $\mathrm{XY}$ ).

Pour chaque animal, l'identification microscopique des hétérochromosomes a porté sur 64 métaphases. $L_{1}$ taille de l'échantillon permet d'affirmer l'absence de chimère ou de mosaiqque sexuelle de fréquence supérieure à $0, I$ avec une probabilité de 0,95 .

\section{III. - MORPHOLOGIE DE L'APPAREIL GÉNITAL}

\section{a) L'individu $X X$}

L'animal $A$ avait à l'autopsie des testicules et épididymes d'allure infantile (fig. I, C et tab1. I). Par ailleurs, l'appareil génital avait une constitution complexe : à côté des éléments normaux de l'appareil génital mâle (canaux déférents, ampoules déférentes, vésicules séminales, prostate, glandes de Cowper, etc.) on pouvait observer au niveau du carrefour urogénital des structures pouvant être des rudiments de tractus femelle et d'autres que nous n'avons pu identifier ( $I, D)$.

\section{b) Les boucs $X Y$}

Les boucs $B, C$ et $D$ présentaient des atteintes bilatérales caractérisées de l'épididyme avec obstruction du canal épididymaire et induration plus ou moins prononcée selon les animaux de l'ensemble de l'organe. Leurs testicules étaient d'un poids inférieur à la normale et présentaient à la dissection des granules de calcification répartis dans tout le parenchyme (tabl. 2). Les boucs $F$ et $G$ présentaient le même type d'atteinte épididymaire que les boucs $B, C$ et $D$ et n'ont été examinés que très sommairement. Les boucs $E$ et $H$ avaient des organes génitaux apparemment normaux pour la saison à laquelle ils ont été examinés.

\section{IV. - ACTIVITÉ TESTICULAIRE}

a) Une image de la production des androgènes testiculaires a été donnée par la teneur en testostérone du sang périphérique au moment de l'abattage des boucs $A, B, C, D$ et $E$ (tabl. 2).

L'individu $\mathrm{XX}(A)$ a une concentration très basse, à la limite de la sensibilité de la techique de dosage.

Les boucs $B, C, D$ et $E$, de caryotype $\mathrm{XY}$, ont des concentrations sanguines de testostérone qui se situent dans les limites des variations individuelles saisonnières normales chez les petits ruminants (ATTAL, non publié).

Planche 2. - Histologie testiculaire des boucs $A, B, C$ et $E$.

A : Animal $\mathrm{XX}(A)$ : tubes séminifères infantiles dans lesquels on ne voit que des cellules somatiques précurseurs des cellules de Sertoli.

$\mathrm{B}$ et $\mathrm{C}:$ Animaux $B$ et $C$ de caryotype XY : tubes séminifères actifs et "vides ". Élimination de cellules dans la lumière des tubes.

$\mathrm{D}$ : Animal $E$ : spermatogenèse active. Noter cependant l'élimination de cellules germinales dans la lumière de certains tubes. 
TABLEAU 2

Poids des testicules et épididymes, concentration en testostérone dans le sang périphérique et histologie testiculaive des boucs stériles A, B, C, D et E

\begin{tabular}{|c|c|c|c|c|c|c|c|}
\hline \multirow{2}{*}{ Boucs } & \multirow{2}{*}{$\begin{array}{l}\text { Sexe } \\
\text { moyen } \\
\text { somique }\end{array}$} & \multirow{2}{*}{$\begin{array}{c}\begin{array}{c}\text { Poids } \\
\text { chromo- } \\
\text { du testicule } \\
(\mathrm{g})\end{array} \\
=\frac{\mathrm{TD}+\mathrm{TG}}{2}\end{array}$} & \multirow{2}{*}{$\begin{array}{l}\text { Poids } \\
\text { moyen } \\
\text { de l'épi- } \\
\text { didyme } \\
\text { (g) }\end{array}$} & \multirow{2}{*}{$\begin{array}{c}\text { Testo- } \\
\text { stérone } \\
\text { mg/ro ml } \\
\text { de plasma } \\
\text { sanguin }\end{array}$} & \multicolumn{2}{|c|}{$\begin{array}{c}\text { Diamètres } \\
\text { tubes } \\
\text { séminifères } \\
(u)\end{array}$} & \multirow{2}{*}{ Spermatogenèse } \\
\hline & & & & & Actifs & Vides & \\
\hline$A$ & $\mathrm{xx}$ & $4, I$ & 6,7 & $0,7^{*}$ & - & 66 & $\begin{array}{l}\text { Tubes très contournés, } \\
\text { pratiquement pas de } \\
\text { cellules germinales, as- } \\
\text { pect infantile, aucune } \\
\text { activité spermatogé- } \\
\text { nétique }\end{array}$ \\
\hline$B$ & $\mathrm{XY}$ & 55,5 & 14,0 & 2,8 & I39 & r 38 & $\begin{array}{l}\text { Grandes zones de paren- } \\
\text { chyme testiculaire avec } \\
\text { tubes vacuolisés pres- } \\
\text { que vides où la sper- } \\
\text { matogenèse avorte en } \\
\text { cours de prophase méi- } \\
\text { otique. Quelques tubes } \\
\text { actifs avec production } \\
\text { de spermatozoides mais } \\
\text { rendements cellulaires } \\
\text { subnormaux }\end{array}$ \\
\hline C & $\mathrm{XY}$ & 60,5 & 24,0 & 8,2 & I 75 & I 37 & $\begin{array}{l}\text { Même allure que B mais } \\
\text { un peu plus de tubes } \\
\text { actifs. Nombreuses cel- } \\
\text { lules germinales imma- } \\
\text { tures desquamées dans } \\
\text { la lumière des tubes }\end{array}$ \\
\hline$D$ & $\mathrm{XY}$ & 78,7 & 24,5 & 14,9 & $\mathrm{I} 64$ & I39 & $\begin{array}{l}\text { Même allure que } B \text { et } C \\
\text { mais un peu plus actif } \\
\text { que } C\end{array}$ \\
\hline$E$ & $\mathrm{XY}$ & I I 7,0 & $2 \mathrm{I}, 8$ & 2,7 & 227 & - & $\begin{array}{l}\text { Apparemment normale } \\
\text { avec toutefois desqua- } \\
\text { mation de cellules ger- } \\
\text { minales immatures } \\
\text { dans la lumière des } \\
\text { tubes. }\end{array}$ \\
\hline
\end{tabular}

* Un contrôle avant abattage avait donné r,8 mg (dosage selon ATral, I969).

b) L'activité spermatogénétique est très variable suivant les animaux (tab1. 2). Elle est inexistante chez l'individu de caryotype femelle (animal $A$ ) où les testicules ont un aspect histologique qui rappelle celui des testicules de l'animal 
nouveau-né. On ne trouve pratiquement pas de cellules germinales dans les tubes séminifères où il ne semble y avoir que des précurseurs des cellules de Sertoli (fig. 2, A).

L'activité spermatogénétique est en relation avec le poids testiculaire chez les boucs XY examinés : les 3 animaux $B, C$ et $D$ dont les testicules sont petits, présentent des zones étendues de parenchyme où les tubes séminifères sont presque vides; la spermatogenèse s'y arrête le plus souvent au cours de la prophase méiotique. A côté de ces zones, on observe cependant un certain nombre de tubes où la spermatogenèse est complète (fig. 2, B et $\mathrm{C}$ ), mais la présence de nombreuses cellules germinales immatures dans la lumière des tubes est l'indication d'un rendement subnormal des processus spermatogénétiques. Même chez l'animal $E$ où la spermatogenèse est apparemment normale dans l'ensemble du testicule (fig. $2, D$ ), on observe des cellules éliminées dans la lumière des tubes séminifères. Peut-être faut-il relier ce phénomène au moment de l'année où ces observations ont été faites (mois de mars), moment qui correspond à la période de repos sexuel des caprins, où, par analogie avec ce que 1'on connaît chez le bélier (ORTAVANT, I958), il pourrait y avoir un moindre rendement spermatogénétique.

\section{V. - PATHOLOGIE MICROBIENNE}

Un testicule de chacun des animaux $A, B, C, D$ et $E$ a été flambé puis broyé stérilement. La purée obtenue a été ensemencée sur plusieurs milieux de culture (W. E., Sérum Dextrose Agar, Tripticase Soy Agar). Tous les examens bactériologiques pratiqués ont été négatifs en ce qui concerne les Brucella et les autres germes poussant sur ces milieux.

Les examens sérologiques de la Brucellose par les réactions de séro-agglutination, de fixation du complément, de Coombs ont été négatives.

\section{DISCUSSION ET CONCLUSION}

La stérilité du " bouc " $\mathrm{XX}(\operatorname{animal} A)$ est due au non fonctionnement de ses gonades, phénomène qui avec l'ensemble des anomalies constatées sur le tractus génital relève certainement de la composition chromosomique (absence de $\mathrm{Y}$ ).

La stérilité des boucs $B, C$ et $D$ est due à l'atteinte pathologique du testicule et de l'épididyme, celle des boucs $F$ et $G$, à celle de l'épididyme sans trace d'infection microbienne comme WEBER (I969) le remarque en Suisse.

Aucune anomalie morphologique ou pathologique observable dans nos conditions d'examen ne peut rendre compte de la stérilité des boucs $E$ et $H$.

L'animal $A$ pourrait être rangé dans la catégorie des hypoplasiques testiculaires et les animaux $B, C, D, F$ et $G$ dans celle des mâles stériles par sténose épididymaire, catégories anatomiques définies déjà par ScHNöNHERR (I954) dans les populations caprines sans cornes. Génétiquement, ils correspondraient à des animaux des 2 sexes homozygotes pour le gène $P$, responsable de l'absence de cornes 
(Soller et al., I969; LAUVERGNe, I969) : $A$ à la catégorie des femelles $P P$ à masculinisation très poussée du tractus génital, les autres à la fraction $b \beta_{2}$ des mâles $P P$ atteints de sténose épididymaire généralisée.

Reçu pour publication en octobre 1969 .

\author{
SUMMARY \\ MORPHOLOGICAL, CARYOLOGICAL, PHYSIOLOGICAL, AND PATHOLOGICAL, \\ EXAMINATION OF STERILE POLLED BILLYGOATS
}

Eight polled billygoats, said to be sterile, were examined. One of them $(A)$, a genetic $\mathrm{XX}$ female, had hypoplasic genitals (Fig. 1). The seven others were caryologic XY males. Two of the genetic male billygoats $(E$ and $H)$ had apparently normal genitals; the others $(B, C, D$, $F$ and $G$ ) had obstructed epididymal ducts and more or less indurated epididymus. Among these, at least three had testis smaller than normal and calcification granules throughout the testicular parenchyma (Table 1) and two were not examined for granules.

The peripheral blood testosterone concentration of $A$ (XX caryotype) was very low, while that of $B, C, D$ and $E$ (XY caryotype) was apparently normal regarding sex and season. Spermatogenetic activity was non-existent in $A$. In $B, C, D$ and $E$, it was about related to the testicular weight (Table 2, fig. 2). With the culture media used, no pathogenic germ was evidenced in the genitals of the billygoats presenting anomalies.

Five of the six animals having genital anomalies might be classed as PP homozygous billygoats in which sterility is said to be due to epididymal stenosis $(B, C, D, F$ and $G)$. The other $(A)$ might be classed as a pseudo-male (Soller et al., 1969; LAUvergne, 1969).

\title{
RÉFÉRENCES BIBLIOGRAPHIQUES
}

Atral J., I969. Levels of testosterone, androstenedione estrone and estradiol-I $7 \beta$ in the testis of fetal sheep. Endocrinology, 85, 280.

LAuvergne J. J., I969. Progrès des connaissances génétiques sur l'intersexualité associée à l'absence de cornes chez la Chèvre d'origine alpine. Ann. Génét. Sél. anim., 1. 403-4I2.

Ortavant R., 1958. Le cycle spermatogénétique chez le bélier. Thèse Doct. Paris, 127 pp., éd. I959; Ann. Zootech., 8, $183-244$ et 271-322.

SCHöNHERR S., 1954. Die Unfruchtbarkeit der Ziegenböcke, ihre Verbreitung, frühzeitige Erkennung und Bekämpfung. Veter. med. Diss. Frei Universität, Berlin.

Soller M., Padeh B., Wysoki M., Ayalon N., r969. Cytogenetics of Saanen goats showing abnormal development of the reproductive tract associated with the dominant gene for polledness. Cyto. genetics, 8, $5 \mathbf{I}-67$.

Weber W., I969. La stérilité des mâles dans les races caprines suisses sans cornes. Ann. Génét. Sél. anim., 1. 379-382. 